\title{
Vertical Microchannels for Microfluidic Multilayer Interconnections in PMMA
}

\author{
Gian-Luca Roth ${ }^{1}$, Cemal Esen ${ }^{2}$ and Ralf Hellmann ${ }^{1}$ \\ ${ }^{1}$ Applied Laser and Photonics Group, University of Applied Sciences Aschaffenburg, \\ Wuerzburger Strasse 45, 63739 Aschaffenburg, Germany \\ E-Mail: Gian-luca.roth@h-ab.de \\ ${ }^{2}$ Applied Laser Technologies, Ruhr-University Bochum, \\ Universitaetsstrasse 150, 44801 Bochum, Germany
}

\begin{abstract}
We report on laser generation of microchannels by focused femtosecond laser pulses inside PMMA bulk material. This process enables direct fabrication of three dimensional microfluidic channel architectures with generally unlimited channel length. Based on nonlinear ultrashort pulse laser absorption of high intensities around the focal volume, a material modification including a refractive index shift is triggered. After laser exposure the channel is created by a gaseous degradation of exposed areas during an annealing process. The 3D channel layout can easily be realized by moving the specimen using 3D motorized stages, allowing freely chosen complex shaped channel architectures. In this report, the creation of inner lying vertical microchannels is examined. In addition to previously shown horizontal channel architectures vertical channels are necessary to fulfill the definition of an internal three-dimensional microfluidic structure and to create interconnections in multilevel lab on chip devices. The cross section and geometry of vertical channels are strongly influenced by laser parameters such as pulse duration, laser power and writing speed. The influence of these process parameters on the channels diameter is determined and parameters are optimized regarding a controllable, stable and reproducible process. The functionality of vertical microchannels as an interconnection between multilayer microfluidic devices is demonstrated using a Rhodamine B solution.
\end{abstract}

DOI: 10.2961/jlmn.2018.03.0003

Keywords: laser materials processing, polymer, ultrashort pulse, femtosecond, microfluidics, hollow microchannels

\section{Introduction}

Microfluidic structures are a core element of common lab on chip (LOC) devices. In combination with photonic, electronic, chemical and biological technologies advanced LOC systems are capable to deliver a micrototalanalysis [1]. Beside inlet and outlet, most current microfluidic systems are primarily two-dimensional [2]. An extension into three dimensional devices would allow higher integration of a broad range of different types of components and would enable the creation of more complex devices and applications [3-6]. To achieve an integration into the third dimension common fabrication methods such as photo lithography or hot embossing being planar 2D technologies are limited, requiring a stacking and bonding of planar processed films. Using glass substrates three dimensional LOCs can be fabricated by selective laser etching (SLE) [7-11]. An ultrashort pulse (USP) laser is used to internally modify material resulting in a higher etching rate in common etchants like $\mathrm{KOH}$ [10] or HF [9]. This process is associated with a high precision and a high surface quality, however, the channel length is limited by etching ratio and the overall processing speed consisting of laser irradiation and etching is quite low. An alternative process to create $3 \mathrm{D}$ structures in transparent substrates is laser direct writing in porous glass [12]. An ultrashort pulse laser is used to ablate 3D microchannels from the lateral edge of a porous glass substrate immersed in water, which is required to wash out debris. The laser process is followed by an annealing step at high temperatures of about $1150{ }^{\circ} \mathrm{C}$ to consolidate the porous of the glass substrate.

Beside glass, transparent polymer materials are a common biocompatible substrate material for LOC devices [13; 14]. Compared to glass, polymers are way cheaper and easier to process using casting and bonding technologies [15; 16]. However, there is no mature wide spread technology to create internal three dimensional channel architectures [17]. Approaches to create 3D-microchannels in transparent polymers are based on the use of high energy pulses [18; 19]. Yet, this method is associated with a low channel quality. An alternative process is presented by Roth et al. [20; 21] using ultrashort laser pulses to initiate an internal material modification by nonlinear absorption. Femtosecond laser modified polymethylmethacrylate (PMMA) exhibits a lower thermal stability as compared to pristine material shown by Baum et al. [22]. In a subsequent process step, laser modified material can be decomposed by an annealing step to create hollow three dimensional internal channel architectures inside of the transparent polymer. The laser generation of microchannels 


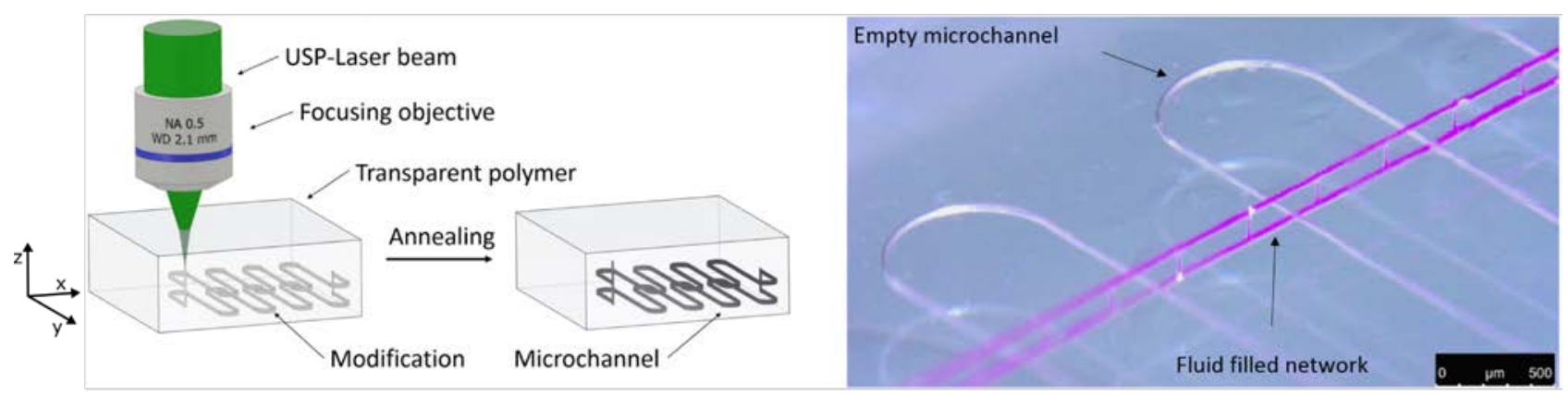

Fig. 1 Scheme of the laser generation of microchannels inside PMMA (left), tilted view on a 3D internal channel architecture using vertical and horizontal microchannels (right). First (top) and third (bottom) layer are connected using vertical microchannels and are filled with a Rhodamine B solution. Crossing meander shaped channels on a second (middle) layer are designed to be empty.

offers the possibility to create 3D microchannels architectures with basically unlimited channel length inside PMMA. As shown by Day and Gu [23] and by Roth et al. [20; 21], cross sections of femtosecond laser generated horizontal channels are strongly influenced by the focusing setup and can be adjusted by laser processing parameters.

By avoiding any etch or solvent processes, this technology is independent of channel length limiting etching selectivity and long processing times. Compared to common ultrashort pulse laser percussion drilling of transparent polymers as shown by Xia et al. [24; 25] internal generation of vertical channels does not suffer under the hole bending phenomenon and is not restricted to the surface of the chip. Furthermore, maximum channel depth is not limited by laser pulse energy but by the working distance of the focusing microscope objective.

In this report, we expand the possibilities to create horizontal channels by studying and demonstrating the creation of circular fully internal vertical channels which are necessary to create multilevel microfluidic channel architectures and to fulfill the definition of a true internal three-dimensional microfluidic structure.

\section{Experimental}

\subsection{Laser System}

We used an ultrashort pulse laser (Light Conversion, Pharos-10-600) with a fundamental wavelength of $1028 \mathrm{~nm}$ having an adjustable pulse duration from 220 fs to 15 ps and a variable repetition rate up to $610 \mathrm{kHz}$. By using a second harmonic generation module, a laser wavelength of $514 \mathrm{~nm}$ can be emitted. The laser beam with a diameter of $4 \mathrm{~mm}$ $\left(1 / \mathrm{e}^{2}\right)$ is focused by a microscope air objectives with a numerical aperture of 0.5 and a working distance of $2.1 \mathrm{~mm}$ (Zeiss, EC Epiplan-Neofluar). Samples are positioned using translation stages with an accuracy of $250 \mathrm{~nm}$ (Aerotech, ANT130-XY) and a repeatability of $75 \mathrm{~nm}$. The focal position is controlled by a nanopositioning z-stage (Aerotech, ANT95-50-L-Z).

\section{$2.2 \quad$ Materials}

Commercially available bulk PMMA sheets (ME303010) with a thickness of $1.1 \mathrm{~mm}$ are used in this study. A CO 2 Laser is applied to cut the sheets into size of
$20 \mathrm{~mm}$ x $20 \mathrm{~mm}$ for the specimens. The polymer exhibits a glass transition temperature of $105^{\circ} \mathrm{C}$. The refractive index $\mathrm{n}$ of the material at the laser wavelength of $514 \mathrm{~nm}$ is 1.4943 at room temperature calculated by Sellmeier equation.

\subsection{Methods and characterization}

Laser direct generation of microchannels consists of two process steps, outlined in Fig. 1 (left). Firstly, the specimen are irradiated by focused femtosecond pulses triggering a nonlinear absorption inside the focal volume. Definable 3D geometry can be created by translating the focal spot in three dimensions inside the bulk. Secondly, the specimen are placed on a hot plate between two glass substrate (thickness $1 \mathrm{~mm}$ ) and annealed at $200^{\circ} \mathrm{C}$ for 30 seconds. The annealing is comparable to the temperature step presented by Day and Gu [23]. Further details can be found in [20]. It is worthwhile to note, that prior annealing no continuous, hollow microchannels are found, i.e. their formation is clearly linked to the annealing process. In order to characterize vertical channel cross sections the treated specimen are ground down to the middle of the created microchannels and polished. An ultrasonic bath is used to remove debris from this process step. Channel geometries are measured by using a reflection and transmission optical microscope (Nikon, 70 Eclipse LVDIA-N). The tilted overview picture is taken with a digital macroscope (Leica, DVM6).

\section{Results and Discussion}

Vertical microchannels are generated inside $1.1 \mathrm{~mm}$ thick PMMA samples with a length of $600 \mu \mathrm{m}$ written in a depth ranging from $200 \mu \mathrm{m}$ to $800 \mu \mathrm{m}$. Please note that contrary to the creation of horizontal microchannels in our case the maximum length of complete vertical microchannels is limited by the thickness of the sample and the working

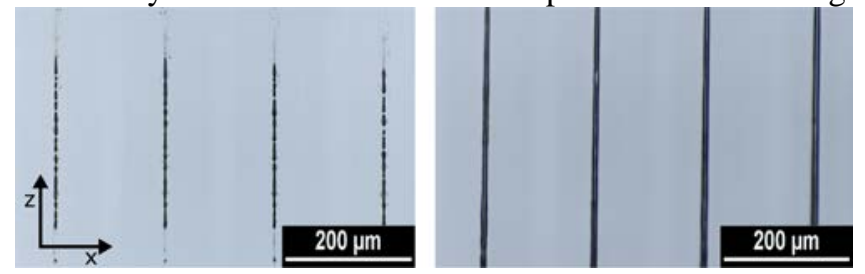

Fig. 2 Side view on vertical microchannels written from bottom to top. Left: pure material modification before annealing triggered solely by the USP laser; Right: opened vertical microchannel after annealing. 


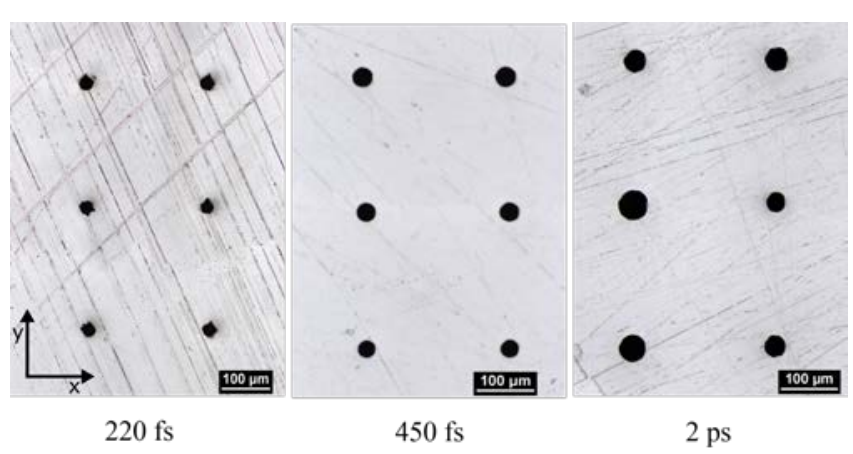

Fig. 3 Cross-section of vertical laser generated microchannels in a $2 \times 3$ array created with different pulse durations.

distance of the objective. The influence of processing parameters laser power, pulse duration writing speed and scanning repetitions on vertical microchannel generation are evaluated. Writing direction is set from bottom to top of the chip to achieve homogeneous and continuous microchannels as depicted in Fig. 2. The creation of a continuous channel, written from top to bottom was not possible and resulted in separated, i.e. not connected spots along the writing lane. The appearance of the initial modification is comparable to modifications observed during the creation of horizontal channels as shown in Ref. [21] and consists of internal bubbles and a refractive index modification. It is worthwhile to note, that channel formation is not related to bubble presence inside the modification. Further process parameters such as wavelength of $514 \mathrm{~nm}$ and a pulse repetition rate of $61 \mathrm{kHz}$ have been determined as suitable in pre-experiments and are used as fixed parameters in this study. Channels are created in arrays of 20 elements per parameter set. Contrary to the creation of horizontal microchannels shown by Roth et al. [20], a pulse duration of $220 \mathrm{fs}$ resulted in a serrated border of the vertical structures. Therefore, the pulse duration was increased to create homogenous, clean and defined channel cross-sections. At a pulse duration above $1 \mathrm{ps}$, channels are generated with a strongly varying diameter. We estimate that a lower nonlinear absorption and a material defect predicated linear absorption is responsible for the varying diameters and therefore the higher standard deviations. As shown in Fig. 3, pulse duration of 450 fs resulted in defined and uniform channels thus this laser pulse duration is set as a fixed parameter for the following study. Compared to horizontal channels, the geometry of the cross section of

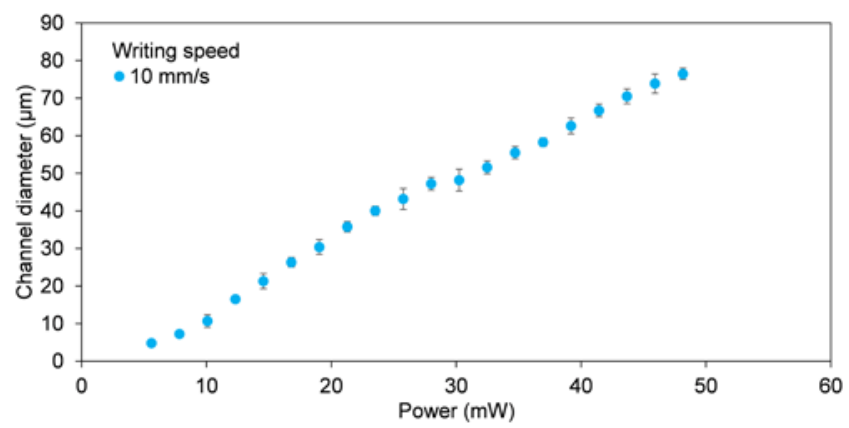

Fig. 4 Impact of laser power on channel diameter for different writing speeds. Error bars include the standard deviation of 20 generated microchannels.

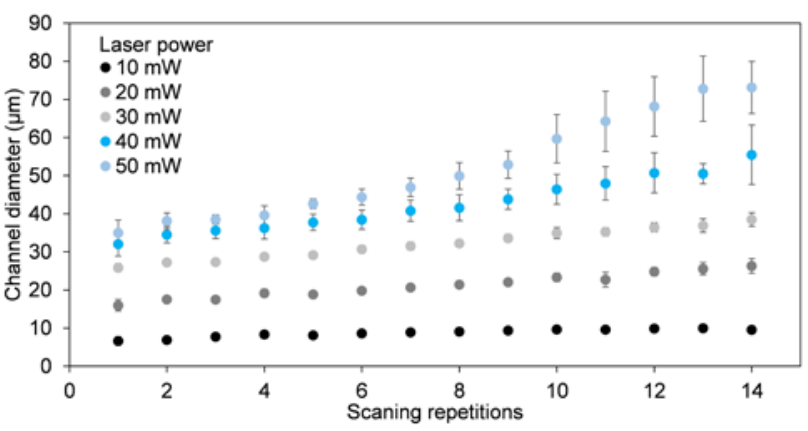

Fig. 5 Influence of scanning repetitions on channel diameter using different laser powers. Error bars include the standard deviation of 20 generated microchannels.

vertical channels is determined by the energy distribution in the focal plane rather than in propagation direction [20], thus enabling a circular cross section. Therefore, channels are classified using its diameter rather than using height and width as it has been done in Ref. 20. Laser power is a suitable process parameter to control and adjust microchannel's diameter. In a range between $5 \mathrm{~mW}$ and $48 \mathrm{~mW}$, the channel diameter can be tuned in a wide range from $7 \mu \mathrm{m}$ to $76 \mu \mathrm{m}$ at a constant low standard deviation and high channel homogeneity. Related data are given in Fig. 4 for a writing speed of $10 \mathrm{~mm} / \mathrm{s}$ using 5 scanning repetitions. The covered diameter range is typical for microfluidic channels [26]. At a laser power above $40 \mathrm{~mW}$, the channel diameter starts to scatter and channel defects can be observed. In comparison to the creation of horizontal microchannels, effective areas of vertical structure are smaller at identical process setup and parameters. For comparison, horizontal microchannels generated at a laser power of $21 \mathrm{~mW}$, using a writing speed of 10 $\mathrm{mm} / \mathrm{s}, 5$ scanning repetitions and a processing depth of 500 $\mu \mathrm{m}$ exhibit a height of $170 \mu \mathrm{m}$ and a width of $70 \mu \mathrm{m}$.

In order to create a reproducible and reliable process, microfluidic structures have to be scanned several times. If only one scanning repetition is used there is a possibility of process failure e.g. no microchannel is generated. In Fig. 5, the influence of the number of scanning repetitions on the diameter of the microchannel for different laser powers is shown. If channels are created with high laser powers, microchannel diameter is significantly enlarged by using a higher number of scanning repetitions. Nevertheless, there is also a larger scattering of the channel diameter.

Quality and diameter of microchannels are also influenced

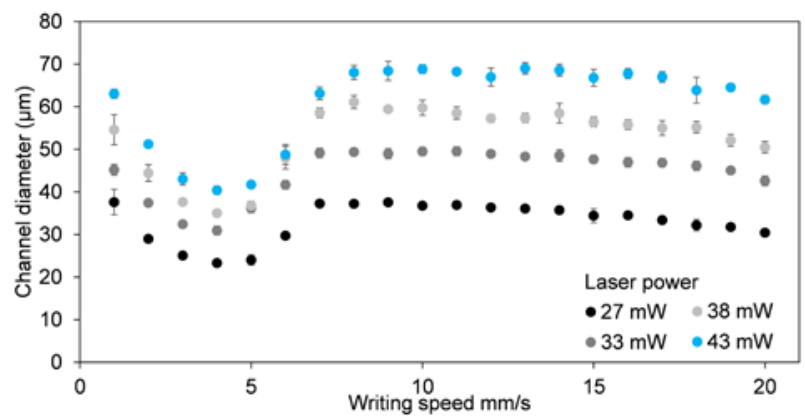

Fig. 6 Influence of writing speed on channel diameter using different laser powers. Error bars include the standard deviation of 20 generated microchannels. 


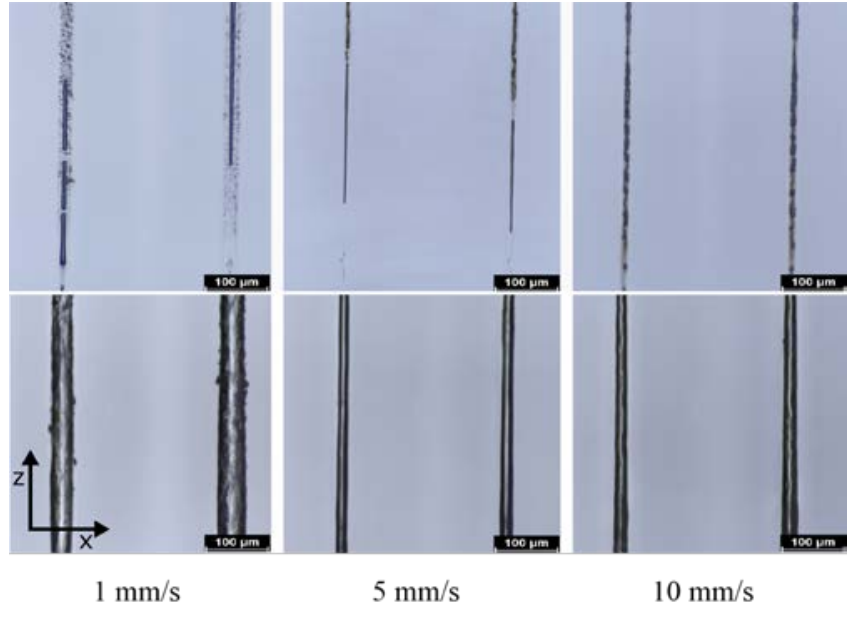

Fig. 7 Side view on vertical microchannels generated with different writing speeds as being modifications before annealing (top row) and as vertical microchannels after annealing (bottom row). Corresponding laser powers are $27 \mathrm{~mW}, 22 \mathrm{~mW}$ and $17 \mathrm{~mW}$ using a writing speed of $1 \mathrm{~mm} / \mathrm{s}, 5 \mathrm{~mm} / \mathrm{s}$ and $10 \mathrm{~mm} / \mathrm{s}$, respectively.

by the writing speed as summarized in Fig. 6 and shown in related transmission microscopy side view pictures given in Fig. 7 before and after annealing. At a writing speed of $1 \mathrm{~mm} / \mathrm{s}$ the heat impact leads to a degeneration of the modified area visible by small black dots inside and outside the writing lane and results in a large and inhomogeneous channel profile. Using a writing speed of $5 \mathrm{~mm} / \mathrm{s}$ a continuous modification can be obtained. Inside the modified track irregular bubbles are observable which are similar to irradiation results by Pätzold et al. [27] writing horizontal waveguides in PMMA and describing non-periodic disruptions along the modification lane. After annealing this modification results in a homogeneous channel profile. Former location of bubbles inside annealed microchannels are not recognizable. It is worthwhile to note, that channel creation is not related to bubble presence, i.e. channels without bubbles along the modified lane can also be transformed into microchannels. In contrast to channel generation at a writing speed of $5 \mathrm{~mm} / \mathrm{s}$, an irregular modification structure is generated using a writing speed of $10 \mathrm{~mm} / \mathrm{s}$. Bubbles appear to be smaller and separated and a distinct coloring along the modified track can be found. After annealing this type of modification results as well as using a laser power below $4 \mathrm{~mm} / \mathrm{s}$ in significantly larger channel diameters as summarized in Fig. 6.

To highlight the functionality of laser generated internal vertical microchannels, a multilevel microfluidic architecture is

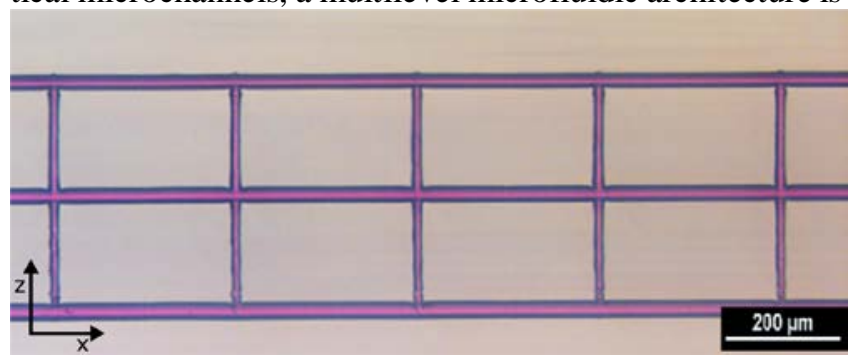

Fig. 8 Side view on multilevel interconnections between three horizontal microfluidic layers using vertical microchannels completely filled with a rhodamine B solution. created. Three levels of horizontal microfluidic channel structures with a vertical spacing of $250 \mu \mathrm{m}$ are filled with a Rhodamine B solution. Vertical channels are written at a speed of $5 \mathrm{~mm} / \mathrm{s}$ with a laser power of $20 \mathrm{~mW}$ and 5 scanning repetitions. The roughness inside the vertical microchannels is measured to be below Ra $0.5 \mu \mathrm{m}$. Different layers are successfully connected by vertical microchannels written from bottom to top as shown in Fig. 8. The fabrication of such a structure starts with the creation of horizontal modifications beginning with the lowest one. Afterwards continuous vertical modifications are written from bottom to top and are annealed in a subsequent step.

\section{Conclusion}

In this study femtosecond laser generation of vertical microchannels inside PMMA with no etching ratio limitations is reported. The formation of microchannels is based on nonlinear absorption around the focal volume triggering a material modification. The modified volume is selectively opened to form the channel by a subsequent annealing process with the microchannel cross-section being orders of magnitude larger than the focal diameter. A processing regime is identified within circular, homogeneous and uniform vertical microchannels inside bulk PMMA are created. Maximum channel length in vertical direction is limited by the sample height and the working distance of the employed focusing objective. The usability of this technology for interconnection of fully internal multilevel microfluidic devices is demonstrating using a Rhodamine $\mathrm{B}$ solution inside a three level channel architecture. The demonstrated process expands the possibilities of laser generation of microchannels inside PMMA.

\section{References}

[1] G. M. Whitesides: Nature, 442, (2006) 368.

[2] P. Abgrall and A.-M. Gue: J. Micromech. Microeng., 17, (2007) R15.

[3] Y. Liao, J. Song, E. Li, Y. Luo, Y. Shen, D. Chen, Y. Cheng, Z. Xu, K. Sugioka and K. Midorikawa: Lab Chip, 12, (2012) 746.

[4] P. Paiè, F. Bragheri, R. M. Vazquez and R. Osellame: Lab Chip, 14, (2014) 1826.

[5] Y. Cheng: Micromachines, 8, (2017) 59.

[6] P. Paiè, F. Bragheri, T. Claude and R. Osellame: Opt. Express, 25, (2017) 7313.

[7] A. Schaap, T. Rohrlack and Y. Bellouard: J. Biophotonics, 5, (2012) 661.

[8] K. Sugioka and Y. Cheng: Appl. Phys. Rev., 1, (2014) 041303.

[9] Y. Bellouard, A. Champion, B. Lenssen, M. Matteucci, A. Schaap, M. Beresna, C. Corbari, M. Gecevicius, P. Kazansky and O. Chappuis: J. Laser Micro/Nanoeng, 7, (2012) ,1.

[10] M. Hermans, J. Gottmann and F. Riedel: J. Laser Micro/Nanoeng., 9, (2014) 126.

[11] S. Kiyama, S. Matsuo, S. Hashimoto and Y. Morihira: J. Phys. Chem. C, 113, (2009) 11560.

[12] Y. Liao and Y. Cheng: Micromachines, 5, (2014) 1106. 
[13] S. Hessler, M. Rüth, C. Sauvant, H.-D. Lemke, B. Schmauss and R. Hellmann: Sens. Actuators, B, 239, (2017) 916.

[14] H. Becker and L. E. Locascio: Talanta, 56, (2002) 267.

[15] H. Becker and C. Gärtner: Electrophoresis, 21, (2000) 12.

[16] P. S. Nunes, P. D. Ohlsson, O. Ordeig and J. P. Kutter: Microfluid. Nanofluid., 9, (2010) 145.

[17] C. Zheng, A. Hu, K. D. Kihm, Q. Ma, R. Li, T. Chen and W. W. Duley: Small, 11, (2015) 25.

[18] C. Zheng, T. Chen, A. Hu, S. Liu and J. Li: Advanced Laser Processing and Manufacturing, (Beijing), P. 1001804.

[19] L. N. D. Kallepalli, V. R. Soma and N. R. Desai: Opt. Eng., 51, (2012) 073402.

[20] G.-L. Roth, C. Esen and R. Hellmann: Opt. Express, 25, (2017) 18442.

[21] G.-L. Roth, C. Esen and R. Hellmann: J. Laser Appl., 30, (2018) 032016.

[22] A. Baum, P. J. Scully, W. Perrie, D. Liu and V. Lucarini: J. Opt. Soc. Am. B, 27, (2010) 107.

[23] D. Day and M. Gu: Opt. Express, 13, (2005) 5939.

[24] B. Xia, L. Jiang, X. Li, X. Yan and Y. Lu: Opt. Express, 23, (2015) 27853.

[25] B. Xia, L. Jiang, X. Li, X. Yan, W. Zhao and Y. Lu: Appl. Phys. A, 119, (2015) 61.

[26] M. Joanicot and A. Ajdari: Science, 309, (2005) 887.

[27] W. M. Pätzold, C. Reinhardt, A. Demircan and U. Morgner: Opt. Lett., 41, (2016) 1269.

(Received: June 23, 2018, Accepted: October 7, 2018) 